\title{
Kompass
}

Nutrition \& Dietetics

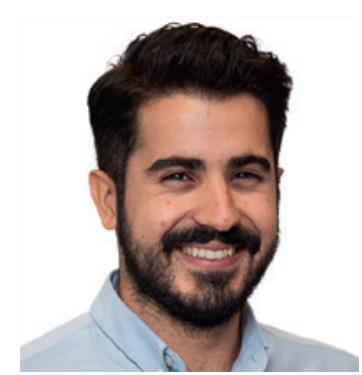

\section{Bariatric Surgery as the Culprit of Malnutrition}

\section{Antonis Vlassopoulos ${ }^{a, b}$}

aDepartment of Food Science \& Human Nutrition, Agricultural University of Athens, Athens, Greece; ${ }^{b}$ Department of Nutritional Sciences \& Dietetics, International Hellenic University, Thessaloniki, Greece

Abstract from Lange J, Königsrainer A: Malnutrition as a complication of bariatric surgery - a clear and present danger? Visc Med 2019; 35:305-311.

\section{Keywords}

Malnutrition · Nutritional status · Obesity · Postoperative monitoring $\cdot$ Sleeve gastrectomy
Abstract
Obesity is a chronic life-threatening disease, and bariatric surgery is the most effective treatment in those patients. The two main operations are laparoscopic sleeve gastrectomy (LSG) and Roux- en-Y gastric bypass (RYGB). LSG carries a smaller risk for nutrition- al deficiencies, while gastric bypass procedures are associated with increased nutritional deficiencies because the procedure is more complex and changes the gastrointestinal anatomy. Recent studies comparing LSG and RYGB have proven that these types of

operation may lead to a similar weight reduction effect but cause different micronutrient deficiencies. Types of malnutrition after bariatric surgery include protein-energy malnutrition and deficiencies of micronutrients, such as iron, folate, vitamin A, and vitamin $B_{12}$. Bariatric patients who do not adhere to the recommended diets are at a greater risk of developing relevant malnutrition. Therefore, life-long postoperative clinical and laboratory monitoring is necessary to diagnose deficiencies of vitamins, trace elements, and minerals and to correct them with supplements. Unfortunately, no standardized aftercare regimes exist for these patients, and the costs for nutritional supplements are paid by the patients themselves.

() 2019 S. Karger AG, Basel 


\section{Knowledge Transfer}

\section{Background}

With at least 2.8 million people dying every year from comorbidities linked to obesity, therapeutic options beyond lifestyle changes are continuing to attract great attention. Data from the longest study on patients who underwent bariatric surgery showed significant improvement in quality of life and reductions in mortality rates from chronic diseases (fig. 1) [1]. Despite positive outcomes, specific types of bariatric surgery are associated with an increased risk of nutritional deficiencies [2].

The latest guidelines published by the German Society for Metabolic \& Bariatric Surgery highlight the need for pre- and postoperative assessments of micronutrient status, especially among women [2] A review by Lange and Königsrainer [3] focused on the topic of

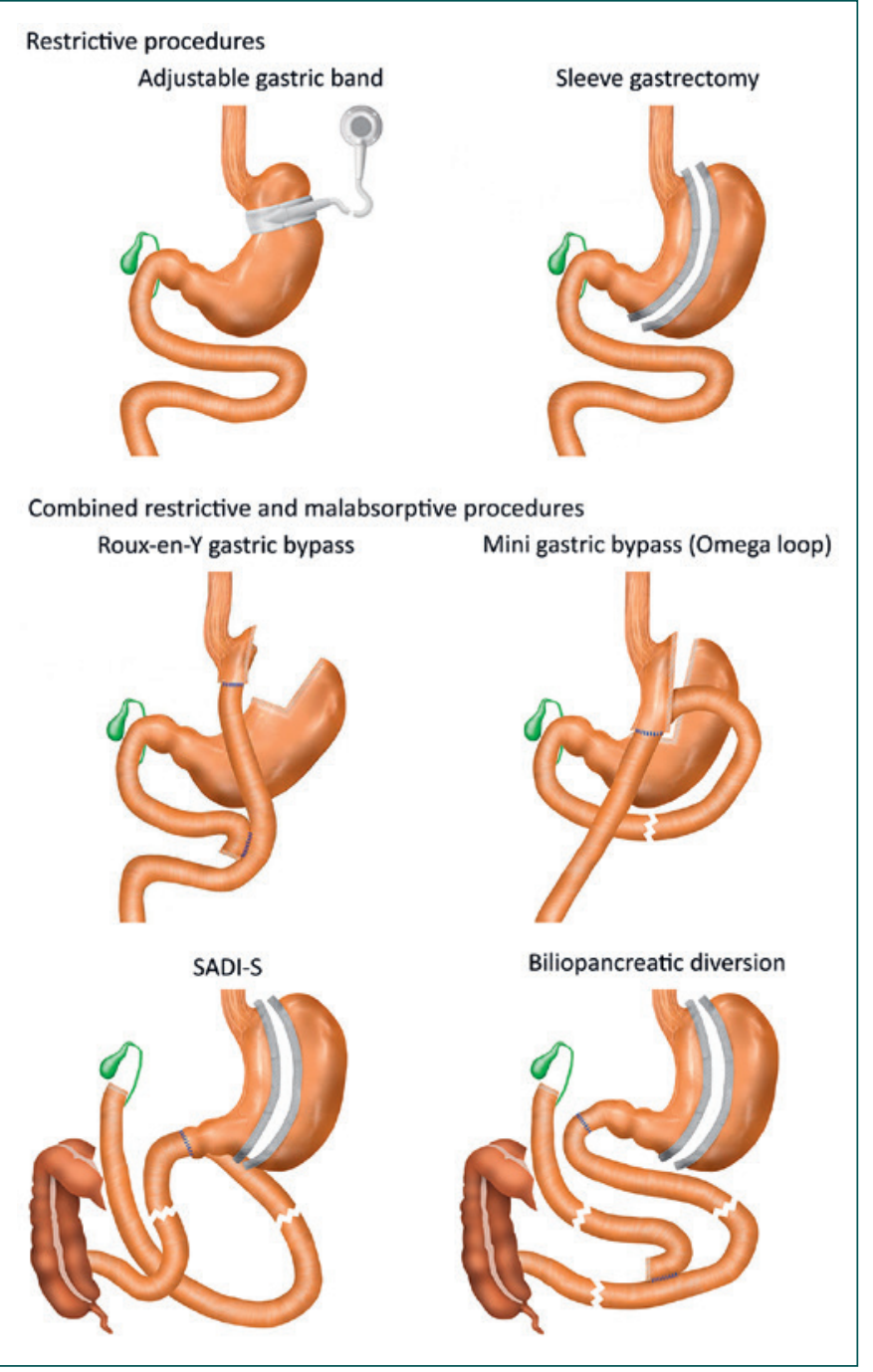

Fig. 1. Commonly performed bariatric surgeries divided into restrictive (adjustable gastric band, sleeve gastrectomy) and malabsorptive (Rouxen-Y gastric bypass, mini gastric bypass, SADI-S (single anastomosis duodenojejunal bypass with sleeve gastrectomy), biliopancreatic diversion) procedures. Image by [3]. malnutrition after bariatric surgery and identified iron, folate, vitamin $A$, and vitamin $B_{12}$ as the most common nutrients of concern alongside protein-energy malnutrition. The review highlights the key deficiencies linked with each type of bariatric surgery and provides recommendations for their treatment.

\section{Study Results}

Bariatric surgery is an increasingly common treatment for severe forms of obesity. As a procedure with low mortality rates, bariatric surgery is an effective tool against obesity in addition to protecting against cardiovascular disease and lowering cancer mortality. However, the extensive changes in the gastrointestinal anatomy are often linked with an increased risk of postsurgical malnutrition. The review by Lange and Königsrainer identified a higher risk of malnutrition for more complex operations that change the gastrointestinal anatomy significantly [3]. According to the review and the latest German guidelines on the care of patients undergoing bariatric surgery, preoperative screening for micronutrient deficiencies is required, and treatment of these deficiencies prior to surgery is strongly recommended. Patients receiving restrictive surgery are likely to require supplementation for iron and vitamin $B_{12}$ and, to a lesser degree, for zinc, calcium, and vitamin $\mathrm{D}_{3}$. On the other hand, patients undergoing malabsorptive procedures will require lifelong supplementation of many micronutrients. They will also require additional attention to ensure adequate protein intake to avoid developing sarcopenic obesity.

Finally, it is important to highlight the need for annual testing for deficiencies, as the lack of standardized care and the failure of health insurances to cover the cost of supplements create conditions that promote underdiagnosis and suboptimal nutritional care for those patients.

\section{Key Malnutrition Considerations after Bariatric Surgery} 1. In restrictive operations (gastric band and sleeve gastrectomy): Limited impact on metabolic needs, however micronutrient deficiencies are common due to reduced food consumption. Patients should be checked for B vitamin deficiencies, especially $B_{1}$ and $B_{12}$. Symptoms of beriberi may appear and should be treated with intravenous administration of $100 \mathrm{mg}$ vitamin $B_{1}$.

- A quarter of all patients will require regular supplementation for $B_{12}$ (1,000-3,000 $\mu \mathrm{g}$ every 3-6 months; exception: patients with a gastric band).

- Iron deficiency is common in more than half of the patients and is often persistent, with low ferritin levels up to 5 years post operation. Iron deficiency often requires intravenous treatment.

- Zinc, calcium, and vitamin $D_{3}$ supplementation is required in $>$ $25 \%$ of patients

2. In patients undergoing malabsorptive procedures: Malabsorptive procedures are associated with malabsorption and maldigestion of a variety of micronutrients. It is recommended that all patients are screened and treated for deficiencies before undergo- 
ing bariatric surgery.

Recommended supplements for this patient group:

- 1,000-3,000 $\mu \mathrm{g}$ of vitamin $B_{12}$ every 3-6 months.

- 45-60 mg/day of elemental iron (50-100 mg/day for young women).

- Daily administration of vitamin $B_{1}$ at $1.1 \mathrm{mg}$ and $1.2-1.5 \mathrm{mg}$ for women and men, respectively. In the case of neurological disorders linked to thiamine deficiency, intravenous/intramuscular administration of $100 \mathrm{mg}$ thiamine $\left(\mathrm{B}_{1}\right)$ is recommended, often combined with magnesium and other $B$ complex vitamins.

- Vitamin D at a prophylactic dose of 3,000 IU/day. A strong recommendation is made for the screening and treatment of vitamin $\mathrm{D}$ deficiency before surgery.

- Calcium at a minimum dose of 3,000 IU/day; higher doses are required after a biliopancreatic diversion with duodenal switch

- A daily dose of 8-15 mg zinc with a strong recommendation for preoperative screening and annual postoperative assessment of zinc sufficiency for Roux-en-Y gastric bypass and biliopancreatic diversion with duodenal switch.

- A daily dose of $2 \mathrm{mg}$ copper.

- A daily dose of $300 \mathrm{mg}$ magnesium citrate.

- Vitamin A deficiencies (common up to 4 years post operation) should be treated with a daily dose of 5,000-10,000 IU/day.

On a general note, patients following malabsorptive bariatric procedures are advised to secure a $1.5 \mathrm{~g} / \mathrm{kg}$ ideal weight protein intake coupled with exercise for muscle mass maintenance. This is increased to $2.2 \mathrm{~g} / \mathrm{kg} /$ day for obese patients in intensive care units.

\section{General Remarks}

Patients planning bariatric surgery should be screened and treated for micronutrient deficiencies prior to surgery. The majority of patients undergoing malabsorptive procedures will require lifelong supplementation of a range of micronutrients, which creates a cost often not covered by their insurance. Due to the lack of standardized guidelines for care, deficiencies are often overlooked in this population.

\section{Disclosure Statement}

I hereby declare that there are no conflicts of interest with regard to this commentary.

\section{References}

1 Sjöström, L., Narbro, K., Sjöström, C. D., Karason, K., Larsson, B., Wedel, H., .. Carlsson, L. M. S. (2007). Effects of Bariatric Surgery on Mortality in Swedish Obese Subjects. New England Journal of Medicine, 357(8), 741-752. https:// doi.org/10.1056/NEJMoa066254.

2 Aills, L., Blankenship, J., Buffington, C., Furtado, M., \& Parrott, J. (2008, September). ASMBS Allied Health Nutritional Guidelines for the Surgical Weight Loss Patient. Surgery for Obesity and Related Diseases, Vol. 4. https://doi. org/10.1016/j.soard.2008.03.002

3 Lange, J., \& Königsrainer, A. (2019, October 1). Malnutrition as a Complication of Bariatric Surgery - A Clear and Present Danger? Visceral Medicine, Vol. 35, pp. 305-311. https://doi.org/10.1159/000503040

Correspondence to:

Dr. Antonis Vlassopoulos, avlassopoulos@aua.gr 